\title{
Antimutagenic Activity of Some Natural supplements on Ivermectin ge- notoxicity in Lymphocytes of Buffalo
}

\author{
Aida I. El-makawy and Karima F. Mahrous \\ Cell Biology Department, National Research Center, 12622 Dokki, Giza, Egypt
}

\begin{abstract}
Ivermectin is a veterinary anthelminthic drug, highly effective against a number of arthropod and nematode infestations in vertebrates. The literature reported that ivermectin have mutagenic activities. The extensive use of ivermectin in food producing animals can cause potential hazard to humanity by causing gene mutation or chromosomal aberrations. Recently, there have been considerable efforts to search for naturally occurring substances that can inhibit, reverse, retard or prevent mutagenicity. A wide array of substances derived from edibles and medicinal plants reported to possess anticarcinogenic and antimutagenic activities. Therefore, the aim of this study was to evaluate the modulator role of each of garlic, L-carnitine and wheat germ oil (WGO) against genotoxicity of ivermectin in buffalo lymphocyte cultures by using the cytokinesis block micronucleus assay and chromosomal aberrations test as cytogenetic end-points. Results showed that ivermectin induced dose dependent increase in the frequencies of the binucleated lymphocytes with micronuclei as well as the number of micronuclei in lymphocytes of river buffalo, while the number of binucleated lymphocytes significantly decreased. In addition, ivermectin low dose caused nonsignificant increase in the frequency of total chromosomal aberrations in lymphocytes of river buffalo as compared to control. Whereas, in medium and high doses the frequencies of aberrant cells increased at a significant level $(P \leq 0.001)$ than control. Meanwhile, the treatment with the three natural supplements (garlic, L-carnitine, wheat germ oil) in continuous with ivermectin significantly reduced the frequencies of binucleated lymphocytes with micronuclei, the number of micronuclei and the frequencies of total chromosomal aberrations induced by ivermectin tested doses, and increase the number of binucleated lymphocytes. In addition, the results indicated that there were non-significant differences between the modulator role of garlic, L-carnitine or wheat germ oil against the mutagenicity of ivermectin in blood lymphocytes. Finally, we can concluded that regular consumption of natural antioxidants, antimutagenic compounds is inversely related to the risk of cancer, since the free radicals scavenger activities of antioxidants are very important due to the deleterious role of free radicals in foods and in biological systems.
\end{abstract}

Keywords: Ivermectin - Garlic - L-carnitine - Wheat Germ Oil - peripheral blood lymphocytesChromosomal aberrations -Micronucleus formation.

\section{Introduction}

Veterinary medicines used widely to protect animal health, prevent economic loss, and to help ensure a safe food supply. Veterinary medicine active ingredients detected in various environmental media, including surface and groundwater, sugges-ting the potential for indirect human exposure from such residues (Boxall et al., 2006 and Capleton et al., 2006). In addition, the extensive use of veterinary drugs in food producing animals can cause the presence of drugs residues in food; thus, consumers of foods drive from animals exposed to veterinary drug residues (Galer and Monro, 1998). In recent years, there has been increasing concern that veterinary drugs may present a potential hazard to humanity by causing gene mutation or chromosomal aberrations and considered as potential developmental toxicants (Ardito et al., 1996; Mailhes et al., 1997; Crebelli, 2000; Adler et al., 2002 and El-makawy \& Radwan, 2003). Ivermectin is a veterinary anthelminthic drug, highly effective against a number of arthropod and nematode infestations in vertebrates (Grant and Briggs, 1998). Chemically, it is a form of abam-ectin, origi- 
nally isolated from the actino-mycete Streptomyces avermitilis (Tway et al., 1981). The literatures reported that ivermectin have mutagenic activities in bone marrow cells of mice (El-makawy and Radwan, 2003). In addition, Lankas et al. (1989) studied the effects of ivermectin on reproduction and neonatal toxicity in rats, they determined that doses of ivermectin as low as $400 \mu \mathrm{g} / \mathrm{kg} /$ day were toxic to neonatal rats. Recently, there have been considerable efforts to search for naturally occurring substances that can inhibit, reverse, retard or prevent mutagenicity. Nowadays, naturally occurring compounds with the potential antimutagenic and anticarcinogenic effects are of great importance for their prospective use in cancer chemoprevention and treatment (Miadokova et al., 2005 and Shukla \& Kalra, 2007). A wide array of substances derived from edibles and medicinal plants reported to possess anticarcinogenic and antimutagenic activities (Surh et al., 2001). Garlic known for its medicinal qualities and its uses as antibiotic, antithrombotic and antineoplastic agent (Guyonnet et al., 2000). The anticarcinogenic property of garlic has been attributed to its organosulfur constituents out of whom the major one reported is Diallyl Sulfide, which exhibit strong antioxidant properties (Jung et al., 2001; Robert et al., 2001; Wu et al., 2002 and Lohani et al., 2003). In addition, in recent years, dietary supplements such as Lcarnitine reported to influence the development and amelioration of numerous disease states. The biochemical active amino acid Lcarnitine influences fatty acid dependent energy use and prevents oxygen free radicalinduced cellular damage. This antioxidant activity may explain many L-carnitine beneficial effects that do not appear to be directly associated with enhanced fatty acid betaoxidation (Maher, 2001). Recent literatures reported that L-carnitine might modulate DNA damage and cell proliferation (Boerringter et al., 1993; Vanella et al., 2000 and Santoro et al., 2005). Wheat germ considered as a foodstuff interacting with the immune system due to its content in wheat germ agglutinin, a lectin known to influence several immune functions in vivo and in vitro (Kilpatrick, 1999). Ethanolic extract of wheat germ indicated the presence of classes I and
II antioxidants (Krings et al., 2000). Recent research confirms that wheat germ oil can reduce oxidative stress (Alessandri et al., 2006). Wheat germ oil also contains policosanol, a substance that can be helpful in lowering raised blood sugar and/or cholesterol levels, as well as octacosonal, a substance reported to improve human fitness (Irmak and Dunford, 2005). Recently, interest has increased in the occurrence, importance and consequences of potential genotoxic activity of a variety of drugs and chemicals. At the same time, to combat parasite infections in a variety of animals, large doses of therapeutic agents are required (De Silva et al., 1997) and the same antiparasitics chemicals may also have mutagenic effects on the organisms they are designed for protection. To date, for example, no antihelmintic agents shown to be risk-free (Otubanjo and Mosuro, 2001). Therefore, This work aims to evaluate the modular role of each of garlic, L-carnitine and wheat germ oil against genotoxicity induced by ivermectin in river buffalo lymphocytes by using the Cytokinesis block micronucleus and the chromosomal aberrations assay as cytogenetic end-points.

\section{Materials and methods}

\section{Cells used}

The study was carried out using buffalo peripheral lymphocytes from fresh blood samples. Blood samples were taken from five healthy animals for each group, which had no treatment during the last 3 months. Approximately $10 \mathrm{ml}$ of blood was collected into syringes containing sodium heparin as anticoagulant.

\section{Chemicals}

Ivermectin (CAS no 1119-97-7) was purchased from Pfizer-Egypt-S.A.E. - CairoARE. Under license from Pfizer Inc- Corporate- USA. Garlic (CAS no.240/2000) purchased from ATOS pharma, Cairo-Egypt. Lcarnitine (CAS no. 20679/99) and wheat germ oil (CAS no.451/2) purchased from Arab Co. for pharm and medicinal plants (MEPACO) Egypt.

\section{Doses}

The concentrations of ivermectin (250, 500 and $1000 \mu \mathrm{g} / \mathrm{ml})$ were chosen on 
the basis of a cytotoxicity test (Dean and Danford, 1994), eight blood lymphocyte cultures were prepared. The first considered as a control. The other seven cultures were treated with different concentrations of the drug: $1,10,100,200$ and $500 \mu \mathrm{g} / \mathrm{ml}$, and 1 and $2 \mathrm{mg} / \mathrm{ml}$. The mitotic index frequency scored in each of the eight cultures. 1000 $\mu \mathrm{g} / \mathrm{ml}$ is the concentration that reduced the mitotic index to about $50 \%$ and used as the highest dose. The drug doses of 500 and $250 \mu \mathrm{g} / \mathrm{ml}$ were taken as medium and low doses, respectively. Garlic, L-carnitine and wheat germ oil were used at concentration of $20 \mu \mathrm{g} / \mathrm{ml}$.

\section{Cytokinesis block micronucleus assay}

The CBMN assay was carried out using the standard technique proposed by $\mathrm{Fe}$ nech (1993), with slight modifications according to Surralle's et al. (1994). Briefly lymphocyte cultures were set up by adding $0.5 \mathrm{ml}$ of heparinized whole blood to $4.5 \mathrm{ml}$ of RPMI 1640 chromosome medium supplemented with $20 \%$ heat-inactivated fetal calf serum, antibiotics (penicillin and streptomycin) and L-glutamine (all obtained from Gibco, Eragny, France). Lymphocytes were stimulated by $1 \%$ phytohaemagglutinin (PHA, Gibco). Iver-mectin at the three tested concentrations $(250,500$ and $1000 \mu \mathrm{g} / \mathrm{ml}$.) and the different natural products (garlic, L-carnitine and wheat germ oil) at concentration of $20 \mu \mathrm{g} / \mathrm{ml}$ were added to the cultures $24 \mathrm{~h}$ after phytohaemmaglutinin stimulation. Cytochalasin-B (Cyt$B$, Sigma), at a final concentration of 6 $\mu \mathrm{g} / \mathrm{ml}$ was added at $44 \mathrm{~h}$ after the cultures were established, to arrest cytokinesis of dividing cells. This concentration of Cyt-B was selected because it gives a higher percentage of binucleated cells and a lower baseline MN frequency. Binucleated lymphocytes were harvested $72 \mathrm{~h}$ after culture setting. The cells were collected by centrifugation and washed with hypotonic solution $(0.075 \mathrm{M} \mathrm{KCl})$ at room temperature using vortex. Next, the cells were centrifuged, and a methanol/acetic acid $(3: 1 \mathrm{v} / \mathrm{v})$ solution was gently added. This fixation step was repeated twice and the resulting cells were resuspended in a small volume of fixative solution. The cells were spread onto cold slides dipped in 70\% ethyl alcohol. The slides were air-dried. All the slides were coded prior to scoring.

\section{Chromosomal aberrations assay}

For chromosomal aberrations $1 \mathrm{ml}$ of whole heparinized blood was added to $4 \mathrm{ml}$ RPMI [1640 medium, Sigma), containing $20 \%$ fetal calf serum (Sigma), 4\% phytohaem-aglutinin (PHA, Sigma) and streptomycin $(250 \mu \mathrm{g} / \mathrm{ml})$, culturing took place at $38.5 \mathrm{c}$ and lasted for $72 \mathrm{hr}$. After $24 \mathrm{~h}$ from culture initiation, the test chemicals was added. Colchicines (Sigma) at a concentration of $100 \mu \mathrm{g} / \mathrm{ml}$ was added $1 \frac{1}{2} \mathrm{~h}$ before harvesting. Hypotonic performed with $(0.075 \mathrm{M} \mathrm{KCl})$ for $20 \mathrm{~min}$. The cells were fixed with methanol-acetic acid (3-1). Slides were stained with $10 \%$ Giemsa diluted with phosphate buffer (PH 6.8) for 35 min. in each culture 100 good metaphase examined for scoring of chromosomal aberrations.

\section{Statistical analysis}

Data were compared by one-way analysis of variance (ANOVA). Statistical analysis was performed using SPSS for Windows. Multiple comparisons were performed by the least significant difference Duncan 's test. $\mathrm{P} \leq 0: 05$ were considered as the level of significance.

\section{Results}

Table (1) represents the mean values of binucleated lymphocytes, binucleated lymph-ocytes with MN and the number of micron-uclei induced by different drug concentrations with or without natural supplements. Results showed that iverme-ctin induced dose depe-ndent increase in the frequencies of the binucleated lymphocytes with micronuclei as well as the number of micronuclei in lymphocytes of river buffalo, while the number of binucleated lymphocytes significantly decreased. Meanwhile, co- treatment of the different natural supplements with ivermectin significantly reduced the frequencies of binucleated lymphocytes with $\mathrm{MN}$ and micronuclei induced by ivermectin and increase the number of 
binucleated lymphocytes as shown in chart (1).

Tables 2, 3 and 4 represent the mean frequencies of chromosomal aberrations recorded in blood cultures of river buffalo treated with ivermectin at the three-tested concentrations alone or with the natural supplements as well as in control. The structural chromosomal aberrations studied in the present work included chromatid gaps, chromatid breaks, deletions, fragments and centromeric attenuations. In cultures treated with low dose ivermectin, there was nonsignificant increase in the frequency of total chromosomal aberrations as compared to control. Whereas, medium and high doses caused a significant increase in the frequencies of aberrant cells at the level $(P \leq 0.001)$ when compared with control. Meanwhile, the data of this study showed that the cotreatment with the three natural supplements significantly reduced the frequencies of total chromosomal aberrat-ions induced by all tested doses as shown in the tables $(2,3$ and 4 ) and chart (2).

The current results clearly indicated that treatment with garlic, L-carnitine or wheat germ oil showed non-significant decrease in the frequencies of micronuclei and total chromos-omal aberrations when compared with control.

Table (1): Effect of natural products on frequencies of binucleated cells and micronuclei in ivermactin treated lymphocyte cultures of the river buffalo.

\begin{tabular}{|c|c|c|c|c|}
\hline Treatments & $\begin{array}{c}\text { No. of } \\
\text { examined } \\
\text { cells }\end{array}$ & $\begin{array}{l}\text { Binucleated } \\
\text { lymphocytes }\end{array}$ & $\begin{array}{c}\text { Binucleated lym- } \\
\text { phocytes with Mi- } \\
\text { cronuclei }\end{array}$ & Micronuclei \\
\hline Control & 10000 & $98.80 \pm 2.39 \mathrm{~b}$ & $4.20 \pm 0.84 \mathrm{~h}$ & $4.20 \pm 0.84 \mathrm{~h}$ \\
\hline $250 \mu \mathrm{g} / \mathrm{ml}$ ivermectin & 10000 & $89.60 \pm 1.14 \mathrm{~d}$ & $8.60 \pm 1.14 \mathrm{de}$ & $10.80 \pm 0.84 \mathrm{~d}$ \\
\hline $500 \mu \mathrm{gl} / \mathrm{ml}$ ivermectin & 10000 & $80.40 \pm 1.14 \mathrm{e}$ & $13.80 \pm 1.30 \mathrm{~b}$ & $16.20 \pm 1.30 \mathrm{~b}$ \\
\hline $1000 \mu \mathrm{g} / \mathrm{ml}$ ivermectin & 10000 & $61.60 \pm 1.82 \mathrm{~h}$ & $20.40 \pm 2.88 \mathrm{a}$ & $25.20 \pm 3.11 \mathrm{a}$ \\
\hline Garlic $20 \mu \mathrm{g} / \mathrm{ml}$ & 10000 & $108.60 \pm 3.85 \mathrm{a}$ & $3.40 \pm 0.55 \mathrm{~h}$ & $3.40 \pm 0.55 \mathrm{~h}$ \\
\hline $250 \mu \mathrm{g} / \mathrm{ml}$ ivermectin + garlic & 10000 & $99.00 \pm 1.00 \mathrm{~b}$ & $6.40 \pm 1.14 \mathrm{~g}$ & $8.00 \pm 1.00 \mathrm{~g}$ \\
\hline $500 \mu \mathrm{g} / \mathrm{ml}$ ivermectin + garlic & 10000 & $87.40 \pm 7.02 \mathrm{~d}$ & $8.20 \pm 0.84 \mathrm{def}$ & $10.00 \pm 0.71 \mathrm{def}$ \\
\hline $1000 \mu \mathrm{g} / \mathrm{ml}$ ivermectin + garlic & 10000 & $79.60 \pm 1.14 \mathrm{ef}$ & $9.60 \pm 0.55 \mathrm{~d}$ & $12.60 \pm 1.14 \mathrm{c}$ \\
\hline L-carnitine $20 \mu \mathrm{g} / \mathrm{ml}$ & 10000 & $106.20 \pm 3.56 \mathrm{a}$ & $3.80 \pm 0.84 \mathrm{~h}$ & $3.80 \pm 0.84 \mathrm{~h}$ \\
\hline $250 \mu \mathrm{g} / \mathrm{ml}$ ivermectin +l-carnitine & 10000 & $94.00 \pm 1.00 \mathrm{c}$ & $6.80 \pm 0.84 \mathrm{fg}$ & $8.60 \pm 0.89 \mathrm{fg}$ \\
\hline $500 \mu \mathrm{g} / \mathrm{ml}$ ivermectin + l- carnitine & 10000 & $81.40 \pm 1.67 \mathrm{e}$ & $9.00 \pm 1.00 \mathrm{de}$ & $10.40 \pm 1.14 \mathrm{de}$ \\
\hline $1000 \mu \mathrm{g} / \mathrm{ml}$ ivermectin + l-carnitine & 10000 & $76.40 \pm 1.67 \mathrm{f}$ & $11.40 \pm 1.67 \mathrm{c}$ & $12.80 \pm 1.64 \mathrm{c}$ \\
\hline Wheat germ oil $20 \mu \mathrm{g} / \mathrm{ml}$ & 10000 & $99.60 \pm 1.14 \mathrm{~b}$ & $4.00 \pm 0.71 \mathrm{~h}$ & $4.00 \pm 0.71 \mathrm{~h}$ \\
\hline $250 \mu \mathrm{g} / \mathrm{ml}$ ivermectin + wheat germ oil & 10000 & $90.00 \pm 1.41 \mathrm{~d}$ & $7.60 \pm 1.14$ efg & $9.00 \pm 0.71$ efg \\
\hline $500 \mu \mathrm{g} / \mathrm{ml}$ ivermectin + wheat germ oil & 10000 & $80.20 \pm 1.10 \mathrm{e}$ & $9.40 \pm 0.55 \mathrm{~d}$ & $10.80 \pm 0.84 \mathrm{de}$ \\
\hline $1000 \mu \mathrm{g} / \mathrm{ml}$ ivermectin + wheat germ oil & 10000 & $72.40 \pm 2.07 \mathrm{~g}$ & $11.80 \pm 0.84 \mathrm{c}$ & $13.20 \pm 1.30 \mathrm{c}$ \\
\hline
\end{tabular}

Means with different letters within each column are significant at 5\% level. 
Aida I. El-makawy \& Karima F. Mahrous

Table (2): Mean values of different chromosomal aberrations induced by ivermectin with or without garlic in lymphocytes of the river buffalo.

\begin{tabular}{|c|c|c|c|c|c|c|c|c|}
\hline \multirow{2}{*}{$\begin{array}{l}\text { Experimental } \\
\text { Groups }\end{array}$} & \multicolumn{5}{|c|}{ Structural chromosomal aberrations } & \multirow{2}{*}{$\begin{array}{c}\text { Total } \\
\text { excluded } \\
\text { gaps }\end{array}$} & \multirow{2}{*}{$\begin{array}{c}\text { Total } \\
\text { With gaps }\end{array}$} & \multirow{2}{*}{$\begin{array}{l}\text { Poly- } \\
\text { ploidy }\end{array}$} \\
\hline & gaps & breaks & deletions & fragments & $\begin{array}{l}\text { centromeric } \\
\text { attenuations }\end{array}$ & & & \\
\hline Control & $\begin{array}{l}1.80 \mathrm{~cd} \\
\pm \\
0.45\end{array}$ & $\begin{array}{l}0.80 \mathrm{~d} \\
\pm \\
0.45\end{array}$ & $\begin{array}{l}0.00 \mathrm{c} \\
\pm \\
0.00\end{array}$ & $\begin{array}{l}0.00 \mathrm{~b} \\
\pm \\
0.00\end{array}$ & $\begin{array}{l}1.20 \mathrm{~b} \\
\pm \\
0.84\end{array}$ & $\begin{array}{l}2.00 \mathrm{de} \\
\pm \\
0.71\end{array}$ & $\begin{array}{l}3.80 \mathrm{~d} \\
\pm \\
0.84\end{array}$ & $\begin{array}{l}0.00 \mathrm{c} \\
\pm \\
0.00\end{array}$ \\
\hline $\begin{array}{l}250 \mu \mathrm{g} / \mathrm{ml} \\
\text { ivermectin }\end{array}$ & $\begin{array}{l}1.80 \mathrm{~cd} \\
\pm \\
0.45\end{array}$ & $\begin{array}{l}2.00 \mathrm{~b} \\
\pm \\
0.71\end{array}$ & $\begin{array}{l}0.60 \mathrm{bc} \\
\pm \\
0.55\end{array}$ & $\begin{array}{l}0.00 \mathrm{~b} \\
\pm \\
0.00\end{array}$ & $\begin{array}{l}0.60 \mathrm{~cd} \\
\pm \\
0.55\end{array}$ & $\begin{array}{l}3.20 \mathrm{~d} \\
\pm \\
0.84\end{array}$ & $\begin{array}{l}5.00 \mathrm{c} \\
\pm \\
0.71\end{array}$ & $\begin{array}{l}0.20 \mathrm{~b} \\
\pm \\
0.45\end{array}$ \\
\hline $\begin{array}{l}500 \mu \mathrm{g} / \mathrm{ml} \\
\text { ivermectin }\end{array}$ & $\begin{array}{l}3.00 \mathrm{~b} \\
\pm \\
0.71\end{array}$ & $\begin{array}{l}3.20 \mathrm{a} \\
\pm \\
0.45\end{array}$ & $\begin{array}{l}0.60 \mathrm{bc} \\
\pm \\
0.55\end{array}$ & $\begin{array}{l}0.00 \mathrm{~b} \\
\pm \\
0.00\end{array}$ & $\begin{array}{l}1.20 \mathrm{bc} \\
\pm \\
0.45\end{array}$ & $\begin{array}{l}5.00 \mathrm{~b} \\
\pm \\
0.71\end{array}$ & $\begin{array}{l}8.00 \mathrm{~b} \\
\pm \\
0.71\end{array}$ & $\begin{array}{l}0.80 \mathrm{ab} \\
\pm \\
0.84\end{array}$ \\
\hline $\begin{array}{l}1000 \mu \mathrm{g} / \mathrm{ml} \\
\text { ivermectin }\end{array}$ & $\begin{array}{l}5.00 \mathrm{a} \\
\pm \\
0.71\end{array}$ & $\begin{array}{l}3.80 \mathrm{a} \\
\pm \\
0.45\end{array}$ & $\begin{array}{l}1.40 \mathrm{a} \\
\pm \\
0.55\end{array}$ & $\begin{array}{l}0.80 \mathrm{a} \\
\pm \\
0.45\end{array}$ & $\begin{array}{l}2.20 \mathrm{a} \\
\pm \\
0.84\end{array}$ & $\begin{array}{l}8.20 \mathrm{a} \\
\pm \\
0.45\end{array}$ & $\begin{array}{l}13.20 \mathrm{a} \\
\pm \\
0.84\end{array}$ & $\begin{array}{l}1.20 \mathrm{a} \\
\pm \\
0.84\end{array}$ \\
\hline $\begin{array}{c}\text { Garlic } \\
(20 \mu \mathrm{g} / \mathrm{ml})\end{array}$ & $\begin{array}{l}1.40 \mathrm{~d} \\
\pm \\
0.55\end{array}$ & $\begin{array}{l}1.00 \mathrm{~cd} \\
\pm \\
0.00\end{array}$ & $\begin{array}{l}0.00 \mathrm{c} \\
\pm \\
0.00\end{array}$ & $\begin{array}{l}0.00 \mathrm{~b} \\
\pm \\
0.00\end{array}$ & $\begin{array}{l}0.20 \mathrm{~b} \\
\pm \\
0.45\end{array}$ & $\begin{array}{l}1.20 \mathrm{e} \\
\pm \\
0.45\end{array}$ & $\begin{array}{l}2.60 \mathrm{e} \\
\pm \\
0.55\end{array}$ & $\begin{array}{l}0.00 \mathrm{c} \\
\pm \\
0.00\end{array}$ \\
\hline $\begin{array}{l}250 \mu \mathrm{g} / \mathrm{ml} \\
\text { ivermectin } \\
+ \text { garlic }\end{array}$ & $\begin{array}{l}1.20 \mathrm{~d} \\
\pm \\
0.45\end{array}$ & $\begin{array}{l}0.80 \mathrm{~d} \\
\pm \\
0.45\end{array}$ & $\begin{array}{l}0.40 \mathrm{c} \\
\pm \\
0.55\end{array}$ & $\begin{array}{l}0.00 \mathrm{~b} \\
\pm \\
0.00\end{array}$ & $\begin{array}{l}1.00 \mathrm{~b} \\
\pm \\
0.71\end{array}$ & $\begin{array}{l}2.00 \mathrm{de} \\
\pm \\
0.00\end{array}$ & $\begin{array}{l}3.20 \mathrm{de} \\
\pm \\
0.45\end{array}$ & $\begin{array}{l}0.00 \mathrm{c} \\
\pm \\
0.00\end{array}$ \\
\hline $\begin{array}{l}500 \mu \mathrm{g} / \mathrm{ml} \\
\text { ivermectin } \\
+ \text { garlic }\end{array}$ & $\begin{array}{l}2.20 \mathrm{c} \\
\pm \\
0.45\end{array}$ & $\begin{array}{l}1.40 \mathrm{bcd} \\
\pm \\
0.55\end{array}$ & $\begin{array}{l}0.60 \mathrm{bc} \\
\pm \\
0.55\end{array}$ & $\begin{array}{l}0.00 \mathrm{~b} \\
\pm \\
0.00\end{array}$ & $\begin{array}{l}1.00 \mathrm{~b} \\
\pm \\
1.00\end{array}$ & $\begin{array}{l}2.60 \mathrm{~cd} \\
\pm \\
0.89 \\
\end{array}$ & $\begin{array}{l}4.80 \mathrm{c} \\
\pm \\
0.45\end{array}$ & $\begin{array}{l}0.40 \mathrm{bc} \\
\pm \\
0.55\end{array}$ \\
\hline $\begin{array}{l}1000 \mu \mathrm{g} / \mathrm{ml} \\
\text { ivermectin } \\
+ \text { garlic }\end{array}$ & $\begin{array}{l}3.00 \mathrm{~b} \\
\pm \\
0.00\end{array}$ & $\begin{array}{l}1.60 \mathrm{bc} \\
\pm \\
0.55\end{array}$ & $\begin{array}{l}1.20 \mathrm{ab} \\
\pm \\
0.45\end{array}$ & $\begin{array}{l}0.20 \mathrm{~b} \\
\pm \\
0.00\end{array}$ & $\begin{array}{l}1.20 \mathrm{~b} \\
\pm \\
0.45\end{array}$ & $\begin{array}{l}.20 \mathrm{~b} \\
\pm \\
0.45\end{array}$ & $\begin{array}{l}7.20 \mathrm{~b} \\
\pm \\
0.45\end{array}$ & $\begin{array}{l}0.60 \mathrm{abc} \\
\pm \\
0.55\end{array}$ \\
\hline
\end{tabular}

Means with different letters within each column are significant at $5 \%$ level.

Table (3): Mean values of different chromosomal aberrations induced by Ivermectin with or without L-carnitine in lymphocytes of the river buffalo.

\begin{tabular}{|c|c|c|c|c|c|c|c|c|}
\hline \multirow{2}{*}{$\begin{array}{l}\text { Experimental } \\
\text { Groups }\end{array}$} & \multicolumn{5}{|c|}{ Structural chromosomal aberrations } & \multirow{2}{*}{$\begin{array}{l}\text { Total ex- } \\
\text { cluded } \\
\text { gaps }\end{array}$} & \multirow{2}{*}{$\begin{array}{l}\text { Total } \\
\text { With } \\
\text { gaps }\end{array}$} & \multirow[b]{2}{*}{ polyploidy } \\
\hline & gaps & breaks & deletions & fragments & $\begin{array}{l}\text { centromeric } \\
\text { attenuations }\end{array}$ & & & \\
\hline Control & $\begin{array}{l}1.80 \mathrm{~d} \\
\pm \\
0.45 \\
\end{array}$ & $\begin{array}{l}0.80 \mathrm{c} \\
\pm \\
0.45\end{array}$ & $\begin{array}{l}0.00 \mathrm{c} \\
\pm \\
0.00\end{array}$ & $\begin{array}{l}0.00 \mathrm{~b} \\
\pm \\
0.00\end{array}$ & $\begin{array}{l}1.20 \mathrm{~b} \\
\pm \\
0.84\end{array}$ & $\begin{array}{l}2.00 \mathrm{c} \\
\pm \\
0.71\end{array}$ & $\begin{array}{l}3.80 \mathrm{c} \\
\pm \\
0.84 \\
\end{array}$ & $\begin{array}{l}0.00 \mathrm{c} \\
\pm \\
0.00\end{array}$ \\
\hline $\begin{array}{l}250 \mu \mathrm{g} / \mathrm{ml} \\
\text { ivermectin }\end{array}$ & $\begin{array}{l}1.80 \mathrm{~d} \\
\pm \\
0.45\end{array}$ & $\begin{array}{l}2.00 \mathrm{~b} \\
\pm \\
0.71\end{array}$ & $\begin{array}{l}0.60 \mathrm{bc} \\
\pm \\
0.55\end{array}$ & $\begin{array}{l}0.00 \mathrm{~b} \\
\pm \\
0.00\end{array}$ & $\begin{array}{l}0.60 \mathrm{bc} \\
\pm \\
0.55\end{array}$ & $\begin{array}{l}3.20 \mathrm{c} \\
\pm \\
0.84\end{array}$ & $\begin{array}{l}5.00 \mathrm{c} \\
\pm \\
0.71\end{array}$ & $\begin{array}{l}0.20 \mathrm{~b} \\
\pm \\
0.45\end{array}$ \\
\hline $\begin{array}{l}500 \mu \mathrm{g} / \mathrm{ml} \\
\text { ivermectin }\end{array}$ & $\begin{array}{l}3.00 \mathrm{~b} \\
\pm \\
0.71\end{array}$ & $\begin{array}{l}3.20 \mathrm{a} \\
\pm \\
0.45 \\
\end{array}$ & $\begin{array}{l}0.60 \mathrm{bc} \\
\pm \\
0.55\end{array}$ & $\begin{array}{l}0.00 \mathrm{~b} \\
\pm \\
0.00\end{array}$ & $\begin{array}{l}1.20 \mathrm{~b} \\
\pm \\
0.45\end{array}$ & $\begin{array}{l}5.00 \mathrm{~b} \\
\pm \\
0.71 \\
\end{array}$ & $\begin{array}{l}8.00 \mathrm{~b} \\
\pm \\
0.71\end{array}$ & $\begin{array}{l}0.80 \mathrm{ab} \\
\pm \\
0.84\end{array}$ \\
\hline $\begin{array}{l}1000 \mu \mathrm{g} / \mathrm{ml} \\
\text { ivermectin }\end{array}$ & $\begin{array}{l}5.00 \mathrm{a} \\
\pm \\
0.71 \\
\end{array}$ & $\begin{array}{l}3.80 \text { a } \\
\pm \\
0.45 \\
\end{array}$ & $\begin{array}{l}1.40 \mathrm{a} \\
\pm \\
0.55\end{array}$ & $\begin{array}{l}0.80 \mathrm{a} \\
\pm \\
0.45\end{array}$ & $\begin{array}{l}2.20 \mathrm{a} \\
\pm \\
0.84\end{array}$ & $\begin{array}{l}8.20 \mathrm{a} \\
\pm \\
0.45 \\
\end{array}$ & $\begin{array}{l}13.20 \mathrm{a} \\
\pm \\
0.84\end{array}$ & $\begin{array}{l}1.20 \mathrm{a} \\
\pm \\
0.84\end{array}$ \\
\hline $\begin{array}{l}\text { L-carnitine } \\
(20 \mu \mathrm{g} / \mathrm{ml})\end{array}$ & $\begin{array}{l}1.40 \mathrm{~d} \\
\pm \\
0.55 \\
\end{array}$ & $\begin{array}{l}1.00 \mathrm{c} \\
\pm \\
0.00\end{array}$ & $\begin{array}{l}0.00 \mathrm{c} \\
\pm \\
0.00\end{array}$ & $\begin{array}{l}0.00 \mathrm{~b} \\
\pm \\
0.00\end{array}$ & $\begin{array}{l}0.00 \mathrm{c} \\
\pm \\
0.00\end{array}$ & $\begin{array}{l}1.00 \mathrm{~cd} \\
\pm \\
0.71\end{array}$ & $\begin{array}{l}2.40 \mathrm{~d} \\
\pm \\
0.55\end{array}$ & $\begin{array}{l}0.00 \mathrm{c} \\
\pm \\
0.00\end{array}$ \\
\hline $\begin{array}{l}250 \mu \mathrm{g} / \mathrm{ml} \\
\text { ivermectin } \\
+ \text { L-carnitine }\end{array}$ & $\begin{array}{l}1.20 \mathrm{~d} \\
\pm \\
0.45\end{array}$ & $\begin{array}{l}0.80 \mathrm{c} \\
\pm \\
0.45\end{array}$ & $\begin{array}{l}0.40 \mathrm{c} \\
\pm \\
0.55\end{array}$ & $\begin{array}{l}0.00 \mathrm{~b} \\
\pm \\
0.00\end{array}$ & $\begin{array}{l}0.60 \mathrm{bc} \\
\pm \\
0.55\end{array}$ & $\begin{array}{l}1.80 \mathrm{de} \\
\pm \\
0.89\end{array}$ & $\begin{array}{l}3.00 \mathrm{~d} \\
\pm \\
1.00\end{array}$ & $\begin{array}{l}0.00 \mathrm{c} \\
\pm \\
0.00\end{array}$ \\
\hline $\begin{array}{l}500 \mu \mathrm{g} / \mathrm{ml} \\
\text { ivermectin } \\
+ \text { L-carnitine }\end{array}$ & $\begin{array}{l}2.00 \mathrm{~cd} \\
\pm \\
0.71\end{array}$ & $\begin{array}{l}1.40 \mathrm{bc} \\
\pm \\
0.55\end{array}$ & $\begin{array}{l}0.40 \mathrm{c} \\
\pm \\
0.55\end{array}$ & $\begin{array}{l}0.00 \mathrm{~b} \\
\pm \\
0.00\end{array}$ & $\begin{array}{l}0.60 \mathrm{bc} \\
\pm \\
0.55\end{array}$ & $\begin{array}{l}2.40 \mathrm{bc} \\
\pm \\
0.87\end{array}$ & $\begin{array}{l}4.40 \mathrm{c} \\
\pm \\
0.89\end{array}$ & $\begin{array}{l}0.40 \text { bc } \\
\pm \\
0.55\end{array}$ \\
\hline $\begin{array}{l}1000 \mu \mathrm{g} / \mathrm{ml} \\
\text { ivermectin } \\
+ \text { L-carnitine }\end{array}$ & $\begin{array}{l}2.80 \mathrm{bc} \\
\pm \\
0.84\end{array}$ & $\begin{array}{l}1.80 \mathrm{~b} \\
\pm \\
0.45\end{array}$ & $\begin{array}{l}1.20 \mathrm{ab} \\
\pm \\
0.45\end{array}$ & $\begin{array}{l}0.00 \mathrm{~b} \\
\pm \\
0.00\end{array}$ & $\begin{array}{l}1.20 \mathrm{~b} \\
\pm \\
0.45\end{array}$ & $\begin{array}{l}4.20 \mathrm{~b} \\
\pm \\
0.45\end{array}$ & $\begin{array}{l}7.00 \mathrm{~b} \\
\pm \\
0.45\end{array}$ & $\begin{array}{l}0.60 \mathrm{abs} \\
\pm \\
0.55\end{array}$ \\
\hline
\end{tabular}

Means with different letters within each column are significant at $5 \%$ level. 
Table (4): Mean values of different chromosomal aberrations induced by Ivermectin with or without wheat germ oil in lymphocytes of the river buffalo

\begin{tabular}{|c|c|c|c|c|c|c|c|c|}
\hline \multirow{2}{*}{$\begin{array}{l}\text { Treatment } \\
\text { groups }\end{array}$} & \multicolumn{5}{|c|}{ Structural chromosomal aberrations } & \multirow{2}{*}{$\begin{array}{l}\text { Total ex- } \\
\text { cluding } \\
\text { gaps }\end{array}$} & \multirow{2}{*}{$\begin{array}{l}\text { Total } \\
\text { With } \\
\text { gaps }\end{array}$} & \multirow[t]{2}{*}{ polyploidy } \\
\hline & gaps & breaks & deletions & fragments & $\begin{array}{l}\text { centromeric } \\
\text { attenuations }\end{array}$ & & & \\
\hline Control & $\begin{array}{l}.80 \mathrm{~cd} \\
.45\end{array}$ & $\begin{array}{l}0.80 \mathrm{~d} \\
\pm \\
0.45\end{array}$ & $\begin{array}{l}0.00 \mathrm{c} \\
\pm \\
0.00\end{array}$ & $\begin{array}{l}0.00 \mathrm{~b} \\
\pm \\
0.00\end{array}$ & $\begin{array}{l}1.20 \mathrm{bc} \\
\pm \\
0.84\end{array}$ & $\begin{array}{l}2.00 \mathrm{e} \\
\pm \\
0.71\end{array}$ & $\begin{array}{l}3.80 \mathrm{e} \\
\pm \\
0.84\end{array}$ & $\begin{array}{l}0.00 \mathrm{~b} \\
\pm \\
0.00\end{array}$ \\
\hline $\begin{array}{r}250 \mu \mathrm{g} / \mathrm{ml} \\
\text { ivermectin }\end{array}$ & $\begin{array}{l}1.80 \mathrm{~cd} \\
\pm \\
0.45\end{array}$ & $\begin{array}{l}2.00 \mathrm{~b} \\
\pm \\
0.71\end{array}$ & $\begin{array}{l}0.60 \mathrm{bc} \\
\pm \\
0.55\end{array}$ & $\begin{array}{l}0.00 \mathrm{~b} \\
\pm \\
0.00\end{array}$ & $\begin{array}{l}0.60 \mathrm{~cd} \\
\pm \\
0.55\end{array}$ & $\begin{array}{l}3.20 \mathrm{~d} \\
\pm \\
0.84\end{array}$ & $\begin{array}{l}5.00 \mathrm{~d} \\
\pm \\
0.71\end{array}$ & $\begin{array}{l}0.20 \mathrm{~b} \\
\pm \\
0.45\end{array}$ \\
\hline $\begin{array}{l}500 \mu \mathrm{g} / \mathrm{ml} \\
\text { ivermectin }\end{array}$ & $\begin{array}{l}3.00 \mathrm{~b} \\
\pm \\
0.71\end{array}$ & $\begin{array}{l}3.20 \mathrm{a} \\
\pm \\
0.45 \\
\end{array}$ & $\begin{array}{l}0.60 \mathrm{bc} \\
\pm \\
0.55\end{array}$ & $\begin{array}{l}0.00 \mathrm{~b} \\
\pm \\
0.00\end{array}$ & $\begin{array}{l}1.20 \mathrm{bc} \\
\pm \\
0.45\end{array}$ & $\begin{array}{l}5.00 \mathrm{~b} \\
\pm \\
0.71 \\
\end{array}$ & $\begin{array}{l}8.00 \mathrm{~b} \\
\pm \\
0.71\end{array}$ & $\begin{array}{l}0.80 \mathrm{ab} \\
\pm \\
0.84\end{array}$ \\
\hline $\begin{array}{l}1000 \mu \mathrm{g} / \mathrm{ml} \\
\text { ivermectin }\end{array}$ & $\begin{array}{l}5.00 \mathrm{a} \\
\pm \\
0.71\end{array}$ & $\begin{array}{l}3.80 \mathrm{a} \\
\pm \\
0.45\end{array}$ & $\begin{array}{l}1.40 \mathrm{a} \\
\pm \\
0.55\end{array}$ & $\begin{array}{l}0.80 \mathrm{a} \\
\pm \\
0.45\end{array}$ & $\begin{array}{l}2.20 \mathrm{a} \\
\pm \\
0.84\end{array}$ & $\begin{array}{l}8.20 \mathrm{a} \\
\pm \\
0.45\end{array}$ & $\begin{array}{l}13.20 \mathrm{a} \\
\pm \\
0.84\end{array}$ & $\begin{array}{l}1.20 \mathrm{a} \\
\pm \\
0.84\end{array}$ \\
\hline $\begin{array}{l}\text { Wheat germ oil } \\
(20 \mu \mathrm{g} / \mathrm{ml})\end{array}$ & $\begin{array}{l}1.40 \mathrm{~d} \\
\pm \\
0.55\end{array}$ & $\begin{array}{l}1.00 \mathrm{~cd} \\
\pm \\
0.00\end{array}$ & $\begin{array}{l}0.00 \mathrm{c} \\
\pm \\
0.00\end{array}$ & $\begin{array}{l}0.00 \mathrm{~b} \\
\pm \\
0.00\end{array}$ & $\begin{array}{l}0.20 \mathrm{~d} \\
\pm \\
0.45\end{array}$ & $\begin{array}{l}1.20 \mathrm{e} \\
\pm \\
0.45\end{array}$ & $\begin{array}{l}2.60 \mathrm{f} \\
\pm \\
0.55\end{array}$ & $\begin{array}{l}0.00 \mathrm{~b} \\
\pm \\
0.00\end{array}$ \\
\hline $\begin{array}{l}250 \mu \mathrm{g} / \mathrm{ml} \\
\text { ivermectin } \\
+ \text { wheat germ oil }\end{array}$ & $\begin{array}{l}1.60 \mathrm{~d} \\
\pm \\
0.55\end{array}$ & $\begin{array}{l}0.60 \mathrm{~d} \\
\pm \\
0.55\end{array}$ & $\begin{array}{l}0.20 \mathrm{bc} \\
\pm \\
0.45\end{array}$ & $\begin{array}{l}0.00 \mathrm{~b} \\
\pm \\
0.00\end{array}$ & $\begin{array}{l}0.80 \mathrm{~cd} \\
\pm \\
0.45\end{array}$ & $\begin{array}{l}1.60 \mathrm{e} \\
\pm \\
0.89\end{array}$ & $\begin{array}{l}3.20 \text { e f } \\
\pm \\
0.84\end{array}$ & $\begin{array}{l}0.20 \mathrm{~b} \\
\pm \\
0.45\end{array}$ \\
\hline $\begin{array}{l}500 \mu \mathrm{g} / \mathrm{ml} \\
\text { ivermectin } \\
+ \text { wheat germ oil }\end{array}$ & $\begin{array}{l}1.80 \mathrm{~cd} \\
\pm \\
0.45\end{array}$ & $\begin{array}{l}1.60 \mathrm{bc} \\
\pm \\
0.55\end{array}$ & $\begin{array}{l}0.80 \mathrm{~b} \\
\pm \\
0.45\end{array}$ & $\begin{array}{l}0.20 \mathrm{~b} \\
\pm \\
0.45\end{array}$ & $\begin{array}{l}1.00 \mathrm{bcd} \\
\pm \\
0.71\end{array}$ & $\begin{array}{l}3.60 \mathrm{~cd} \\
\pm \\
1.14\end{array}$ & $\begin{array}{l}5.40 \mathrm{~d} \\
\pm \\
1.34\end{array}$ & $\begin{array}{l}0.60 \mathrm{ab} \\
\pm \\
0.55\end{array}$ \\
\hline $\begin{array}{l}1000 \mu \mathrm{g} / \mathrm{ml} \\
\text { ivermectin } \\
+ \text { wheat germ oil } \\
\end{array}$ & $\begin{array}{l}2.40 \mathrm{bc} \\
\pm \\
0.55 \\
\end{array}$ & $\begin{array}{l}1.80 \mathrm{~b} \\
\pm \\
0.45 \\
\end{array}$ & $\begin{array}{l}0.40 \mathrm{bc} \\
\pm \\
0.55\end{array}$ & $\begin{array}{l}0.40 \mathrm{~b} \\
\pm \\
0.55 \\
\end{array}$ & $\begin{array}{l}1.80 \mathrm{ab} \\
\pm \\
0 . .55\end{array}$ & $\begin{array}{l}4.40 \mathrm{bc} \\
\pm \\
0.89 \\
\end{array}$ & $\begin{array}{l}6.80 \mathrm{c} \\
\pm \\
0.85 \\
\end{array}$ & $\begin{array}{l}0.60 \mathrm{~b} \\
\pm \\
0.55\end{array}$ \\
\hline
\end{tabular}

Means with different letters within each column are significant at $5 \%$ level.

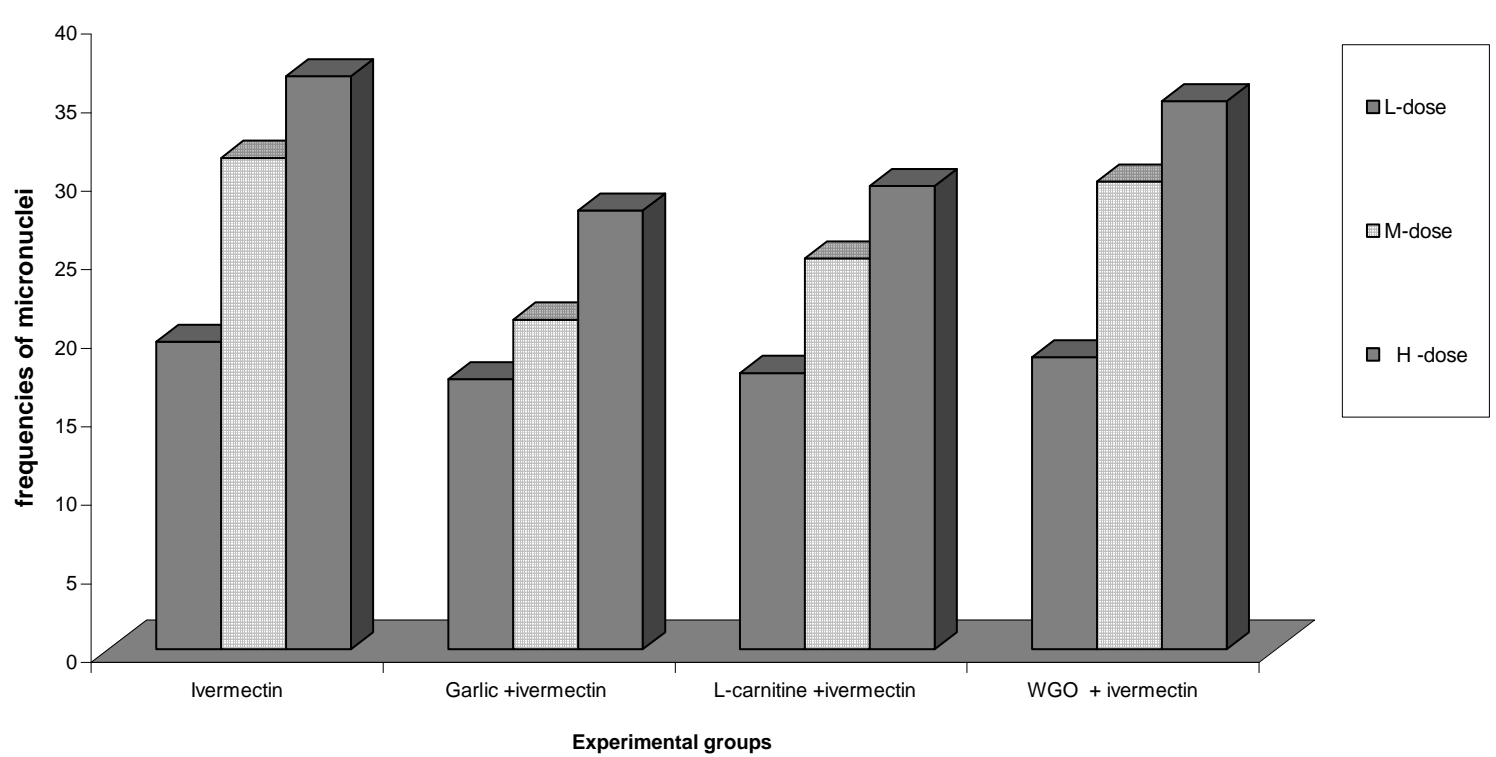




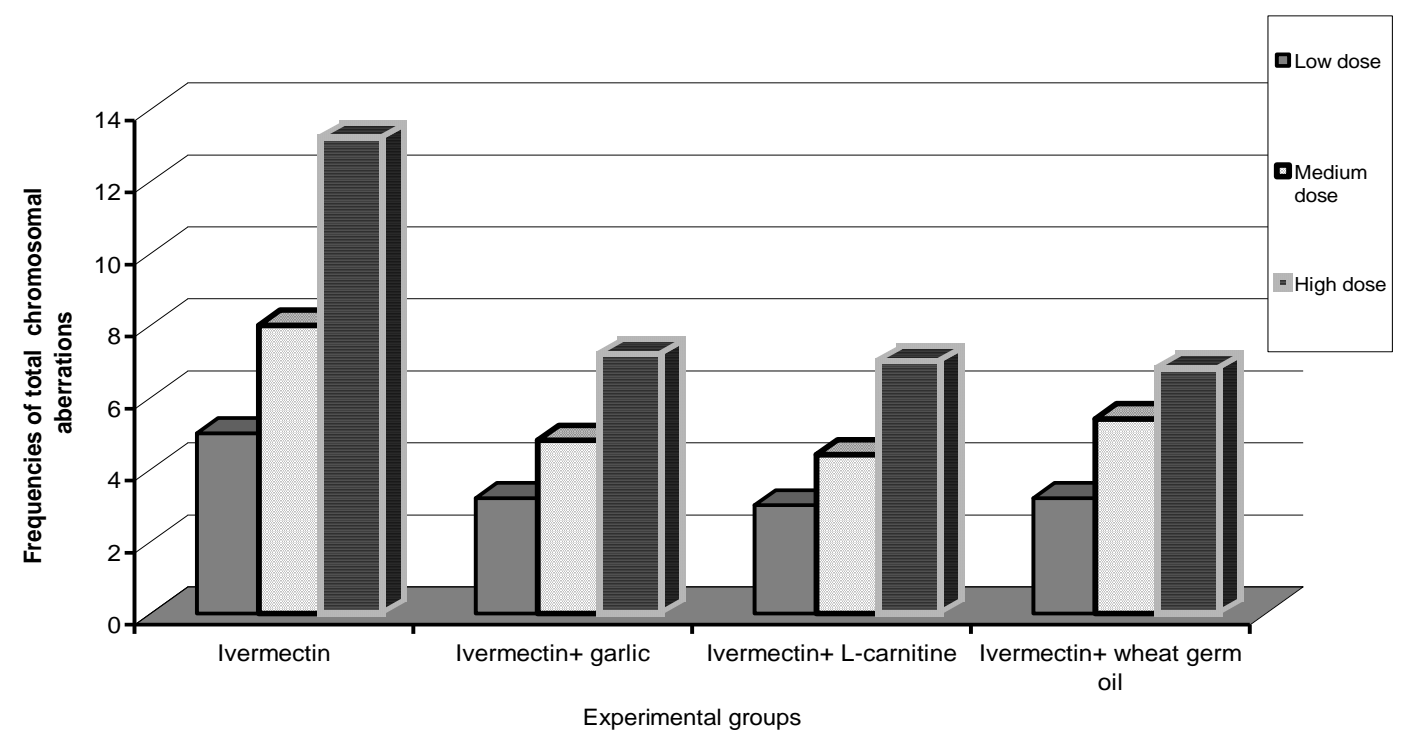

\section{Discussion}

The present investigation was carried out to explore the possible modulator role of garlic, L-carnitine and wheat germ oil on ivermectin genotoxicity in mammalian cells. Ivermectin is a highly active antiparasitic animal drug utilized in a variety of injectable, oral and topical formulations. Residues of this drug may reach the environment through manufacturing and animal wastes and may potentially have effects on terrestrial and aquatic organisms (Bloom and Matheson, 1993). Meanwhile, previous literature reported that ivermectin has mutagenic activities. El-makawy and Radwan, (2003) indicated that ivermectin induced statistically significant increase in the number of micronucleated polychro-matic erythrocytes. This is in agreement with the results of the present study, which showed that ivermectin induced dose dependent significantly increase in the number of binucleated lymphocytes with micronuclei and also the frequencies of total chromosomal aberrations. In addition, the numbers of binucleated lymphocytes showed dose dependent decrease than control. These results revealed that the drug has a cytotoxic effect on the number of cell divisions. As the micronuclei are small chromatincontaining bodies arising from chromosome fragmentation by breaks or deletion, the results of $\mathrm{MN}$ formation confirmed our results of chromosomal aberrations indicating the clastogenic effects of ivermectin. Nowadays, naturally occurring compounds with the potential antimutagenic and anticarcinogenic effects are of great importance for their prospective use in cancer chemoprevention and treatment (Miadokova et al., 2005). A wide array of substances derived from edibles and medicinal plants reported to possess anticarcinogenic and antimutagenic activities (Surh et al., 2001). Natural antioxidants closely related to their bio-functionalities, such as the reduction of chronic diseases like DNA damage, mutagenesis, carcinogenesis and inhibition of pathogenic bacteria growth that is often associated with the termination of free radical propagation in biological systems (Bloom and Matheson, 1993; Covacci et al., 2001 and Zhu et al., 2002). The present investigation revealed the antimutagenic potential of garlic, Lcarnitine and wheat germ oil against the mutagenic effect of ivermectin in the buffalo blood lymph-ocytes. These results were in agreement with Gulcin (2006) who reported that the protective effect of garlic towards cyclophosphamide induced cytotoxic and cytogenetic damage implies as a good marker of its antimutagenic and antineo-plastic activity. In addition, previous literatures showed that continuous daily dietary uptake of raw garlic extract modulated the mutagenicity of sodium arsenate and aflatoxin B (1) (Choudhury et 
al., 1997 and Shukla \& Taneja, 2002). Treatment of male Swiss albino mice with garlic significantly reduced the frequency of micronuclei induced by 7, 12-dimethylbenz-[a]anthracene (DMBA) in bone marrow (Guyonnet et al., 2002). In addition, garlic showed a clear antimut-agenic and immunomodulatory activities on mutagenicity and immunosu-ppression induced by different mutagens (Bhuvaneswari et al., 2004). Chemical analysis has indicated that protective effects of garlic appear to be related to the presence of organosulfur compounds mainly allyl derivatives. Garlic mechanisms to cancer chemopreventive effects include modul-ation in activity of several metabolizing enzymes that activate and detoxify carcinogens and inhibit DNA adduct formation, antioxidative properties, regulation of cell proliferation, apoptosis and immune responses (Shukla and Kalra, 2007). Meanwhile, Barta et al. (2006) demonstrated that L-carnitine treatment decreased DNA damage induced with fumonisin B1 in liver and spleen of rats. In addition, Atroshi et al. (1999) concluded that L-carnitine might act as positive modulator of cytotoxic anticancer agents. In addition, Santoro et al. (2005) stated that L-carnitine reduced hydrogen peroxide formation which induces the chromosomal aberrations. Wheat germ oil posses an antioxidative potential that may protect the body against $\mathrm{He}-\mathrm{Ne}$ laser irradiation due to the amelioration of oxidative stress of free radicals (EL-makawy and El-Ashmaoui, 2003). On the same time, (Omran, 2006 and Zhu et al., 2006) investigated the antioxidant and free radical-scavenging activities of wheat germ oil by employing several in vitro assay systems, including the linoleic acid emulsion model system, 1,1diphenyl-2-picrylhydrazyl (DPPH)/ superoxide/hydroxyl radical-scavenging, reducing power, and ferrous ion-chelating activity. WGO showed scavenging activity against free radicals such as DPPH, superoxide, and hydroxyl radicals. These previous findings suggested the protective role of the used natural supplements and confirmed the report of Zhu et al. (2006) in which they suggested that a diet containing even low levels of different naturally occu-rring compounds is effective in exerting antigenotoxic effects by modulating oxidative stress. In conclusion, the current results revealed that ivermectin induced genotoxic effect in the lympho-cytes of the buffalo, included, increase in the number of micronuclei and chromo-somal abnorm-alities, decrease in the number of binuc-leated lymphocytes. Garlic, L-carnitine and wheat germ oil proved to have antimut-agenic effect against iverm-ectin mutagen-icity. Their modulator role may be attribu-ted to the ability to scavange free radicals and protection of the cell membranes from lipid peroxidation. Generally, the modulator role of these compounds can be explained by their mech-anism in enhancing cellular antioxidant activity by free radical scavenging and augmentation of endogenous antioxidants via prevention of GSH depletion and alteration of GSH dependent enzymes activity and/or their gene expression (Sener et al., 2004; Dokmeci, 2005; Dokmeci et al., 2005 and Sabayan et al., 2007).

\section{References}

1. Adler I. D, Schmid T E. and Baumgartner A (2002): Induction of aneuploidy in male mouse germ cells detected by the sperm-FISH assay: a review of the present data base .Mut. Res., 504(1-2): 173-182.

2. Alessandri C, Pignatelli P, Loffredo L, Lenti L, Del Ben M, Carnevale R, Perrone A, Ferro D, Angelico F. and Violi F. (2006): Alpha-linolenic acid-rich wheat germ oil decreases oxidative stress and CD40 ligand in patients with mild hypercholesterolemia. Arterioscler Thromb. Vasc. Biol., 26(11): 2577-2578

3. Ardito G, Bramanti B, Bigatti P, Lamberti L, and Dolara P. (1996): Cytogenetic effect of thiabendazole and diphenylammine on cultured human lymphocytes; sister chromatid exchanges and cell cycle delay. Boll. Soc. Ital. Biol. Sper., 72 (5-6) :171-178.

4. Atroshi F, Rizzo FA, Biese I, Veijalainen P, Saloniemi H, Sankari S. and Andersson K. (1999): Fumonisin B1-induced DNA damage in rat liver and spleen: effects of pretreatment with coenzyme Q10, Lcarnitine, alpha-tocopherol and selenium. Pharmacol Res. 40(6): 459-467.

5. Barta I, Smerak P, Polivkova Z, Sestakova H, Langova M, Turek 
B. and.Bartova J. (2006): Current trends and perspectives in nutrition and cancer prevention. Neoplasma, 53 (1): 19-25.

6. Bhuvaneswari V, Velmurugan B, Abraham SK and Nagini $S$ (2004): Tomato and garlic by gavage modulate $7, \quad 12$ dimethylbenz[a]anthraceneinduced genotoxicity and oxidative stress in mice. Braz. J. Med. Biol. Res., 37(7):1029-1034.

7. Bloom R A and Matheson J C ( 1993): Environmental assessment of avermectins by the US Food and Drug Administration. Vet. Parasitol., 48(1-4): 281-294.

8. Boerringter M E, Franceschi C, ArrigoniMartelli E, Wei J and Vijg JY (1993): The effect of L-carnitine and acetylL-carnitine on the disappearance of DNA single-strand breaks in human peripheral blood lymphocytes, Carcinogenesis, 14: 2131-2136.

9. Boxall A B, Johnson B P, Smith E J, Sinclair CJ, Stutt E and Levy L S (2006): Uptake of veterinary medicines from soils into plants, J. Agric. Food Chem., 54(6): 2288-2297.

10. Capleton AC, Courage CP, Rumsby CP, Holmes P, Stutt E, Boxall AB and Levy LS(2006): Prioritising veterinary medicines according to their potential indirect human exposure and toxicity profile. Toxicol. Lett. 163(3): 213-223.

11. Choudhury A R, Das T, Sharma A and Talukder G (1997): Inhibition of clastogenic effects of arsenic through continued oral administration of garlic extract in mice in vivo. Mut. Res., 392: 237-242.

12. Covacci V, Torsello A, Palozza P, Sgambato A, Romano G, Boninscgna A, Cittadini A and Wolf EL (2001): DNA oxidative damage during differentiation of $\mathrm{HL}$ 60 human promyelocytic leukemia cells. Chemical Research in Toxicology, 14: 1492-1497.

13. Crebelli R (2000): Thershold- mediated mechanisms in mutagenesis implications in the classification and regulation of chemical mutagens. Mut. Res., 464 (1):129-135.

14. Dean BJ and Danford N (1994): Assays for the detection of chemically-induced chromosome damage in cultured mammalian cells. Cited from: Sahar A. and Othman E. (2003): Clastogenic effects of the fasciolicide drug fasinex on river buffalo lymphocyte cultures in vitro. Mut. Res., 541: 115-121
15. De Silva N, Guyatt $H$ and Bundy $D$ (1997): Anthelmintics. A comparative review of their clinical pharmacology. Drugs, 53(5): 769-788.

16. Dokmeci D (2005): Oxidative stress, male infertility and the role of carnitines. Folia. Med. , 47: 26-30.

17. Dokmeci D, Akpolat M, Aydogdu N, Doganay L and Turan FN (2005): Lcarnitine inhibits ethanol-induced gastric mucosal injury in rats. Pharmacol. Res., 57: 481-488.

18. EL-makawy A and El-Ashmaoui HM (2003): Evaluation of the protective activity of beta-carotene and L-carnitine on doxorubicin-induced genotoxicity in rats. Arab. J. Biotech., 6(2): 289-296.

19. El-makawy A and Radwan HA (2003): Micronuclei induction by some anthelm-intic drugs in male mice. Sc.J.Az. Med.Fac. (Girls), 24(1): 759-769.

20. Fenech M (1993): The cytokinesis micro-nucleus technique: a detailed description of the method and its application to genoto-xicity studies in human populations. Mut. Res., 285:35-44

21. Galer D M and Monro AM (1998): Veterinary drugs no longer need testing for carcinogenicity in rodent bioassays, Regulatory Toxicology and Pharmacology, 28(2):115-123

22. Grant A. and Briggs A.D. (1998): Use of ivermectin in marine fish farms: some concerns. Mar.Pollut.Bull., 36 (8):566-568.

23. Gulcin I (2006): Antioxidant and antiradical activities of L-carnitine, Life Sci., 78(8):803-811.

24. Guyonnet D., Belloir C., Suscheter M., Siess M.H. and Le Bon A. M. (2000): Liver subcellular fractions from rats treated by organosulfur compounds from Allium modulate mutagen activation. Res. ; 466(1): 17-26.

25. Guyonnet D., Belloir C., Suschetet M., Siess M. H. and Le Bon A. M. (2002): Mechanisms of protection against aflatoxin $\mathrm{B}_{1}$ genotoxicity in rats treated by organosulfur compounds from garlic Carcinogenesis, 23 (8): 1335-1341

26. Irrmak $S$ and Dunford NT (2005): Policosanol contents and compositions of wheat varieties. J. Agric. Food Chem., 53 (14): 5583-5586.

27. Jung MY, Kwon SK. and Moon A (2001): Chemopreventive allylthiopyridazine derivatives induce apoptosis in SK- 
Hep-1 hepatocarcinoma cells through a caspase-3-dependent mechanism. Eur. J. Cancer., 37(16): 2104-2110.

28. Kilpatrick DC (1999): Immunological aspects of the potential role of diatary carbohydrates and lectins in human health. Eur. J. Nutr., 38: 107-117.

29. Krings U, El-Saharty Y S , El-Zeany B A, Pabel $B$ and Berger RG (2000): Antioxidant activity of extracts from roasted wheat germ. Food chemistry, 71(1): 91-95.

30. Lankas G R, Minsker DH and Robertson RT (1989): Effects of ivermectin on reproduction and neonatal toxicity in rats. Food Chem. Toxicol., 27(8): 523-529.

31. Lohani M, Yadav S, Schiffmann $D$ and Rahman $Q$ (2003): Diallylsulfide attenuates asbestosinduced genotoxicity. Toxicol. Lett. , 143(1): 45-50.

32. Maher TJ (2001): L-Carnitine: continuing education module. Natural Healing Track, 1 -8. Cited from: EL-makawy A. and ElAshmaoui H.M. (2003): Evaluation of the protective activity of beta-carotene and Lcarnitine on doxorubicin-induced genotoxicity in Rats. Arab. J. Biotech., 6(2): 289296.

33. Mailhes J B, Young D, Aardema M J. and London $S$ N (1997): Thiabendazoleinduced cytogenetic abnormalities in mouse oocytes. Envron. Mol. Mutagen., 29(4): 367-371.

34. Miadokova E, Svidova S, Vlckova V, Duhova V, Prazmariova E, Tothova K, Nadova S, Kogan G and Rauko $P$ (2005): The role of natural biopolymers in genotoxicity of mutagens/carcinogens elimination. Biomed. Pap. Med. Fac. Univ. Palacky. Olomouc. Czech. Repub., 149(2): 493-496.

35. Otubanjo $O A$ and Mosuro A A (2001): An in vivo of induction of abnormal sperm morphology by some anthelmintic drugs in mice. Mutation Research, 97 (12):131:138.

36. Robert V, Mouille B, Mayeur C, Michaud $M$ and Blachier $F$ (2001): Effects of the garlic compound diallyl disulfide on the metabolism, adherence and cell cycle of HT-29 colon carcinoma cells: evidence of sensitive and resistant sub-populations. Carcinogenesis, 22 (8): 1155-1161.

37. Sabayan B, Foroughinia $\mathbf{F}$ and Chohedry A (2007): A postulated role of garlic organosulfur compounds in prevention of valproic acid hepatotoxicity. Medical Hypotheses, 68(3): 512-514.

38. Santoro A, Lioi M B, Monfregola J, Salzano S., Barbieri R. and Ursini M.V. (2005): L-carnitine protects mammalian cells from chromosomal aberrations but not from inhibition of cell proliferation induced by hydrogen peroxide,Mut.Res.,587:16-25.

39. Sener G., Paskaloglu K., Satiroglu H., Alican I., Kacmaz A. and Sakarcan A. (2004): L-carnitine ameliorates oxidative damage due to chronic renal failure in rats. J. Cardiovasc. Pharmacol., 43:698-705.

40. Shukla Y and Kalra N.(2007): Cancer chemoprevention with garlic and its constituents. Cancer Lett. , 247(2): 167-181.

41. Shukla Y. and Taneja P. (2002): Antimutagenic effects of garlic extract on chromosomal aberrations.,Cancer Letters, 176: 3136.

42. Surh Y J, Lee JY and Keum YS (2001): Molecular mechanisms underlying antitumor promoting activities of heat processed panax ginseng. J. Korean Med. Sci., 16: 538-541.

43. Surralle's J, Antoccia A, Creus A, Degrassi F, Peris F, Tanzarella C, Xamena $N$ and Marcos R (1994): The effects of cytochalasin-B concentration on the frequency of micronuclei induced by four standard mutagens. Mutagenesis, 9: 347353.

44. Tway T. C., Wood J. J. and Downing G.V. (1981): Determination of ivermectin in cattle and sheep tissues using highperformance liquid chromatography with fluorescence detection. J. Agric. Food Chem., 29(5): 1059-1063.

45. Vanella A, Russo A, Acquaviva R, Campisi A, Di Giacomo C, Sorrenti V and Barcellona ML (2000): 1PropionyL-carnitine as superoxide scavenger, antioxidant, and cleavage protector, Cell Biol. Toxicol., 16: 99-104.

46. Wu C C, Sheen LY , Chen H.W , Kuo WW, Tsai SJ and Lii CK (2002): Differential effects of garlic oil and its three major organosulfur components on the hepatic detoxification system in rats. J. Agric. Food Chem., 50(2): 378-383.

47. Zhu K, Zhou $H$ and Qian $H$ (2006): Antioxidant and free radical-scavenging activities of wheat germ protein hydrolysates (WGPH) prepared with alcalase. Process Biochemistry, 41(6): 1296-1302.

48. Zhu QY, Hackman R. M, Ensunsa J I, Holt $R$ R and Keen C L (2002): Antioxidative activities of oolong tea. J. Agri- 


\section{التأثير المضاد للطفرات لبعض المكملات الطبيعية ضد التسمم الوراثى

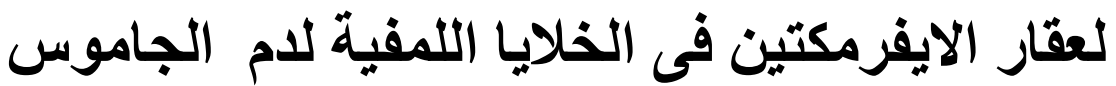

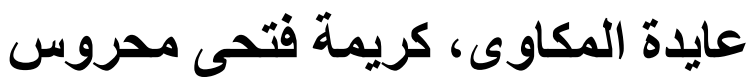

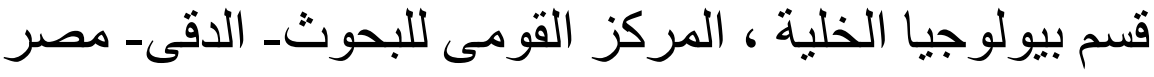

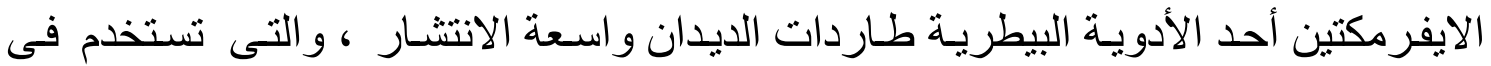

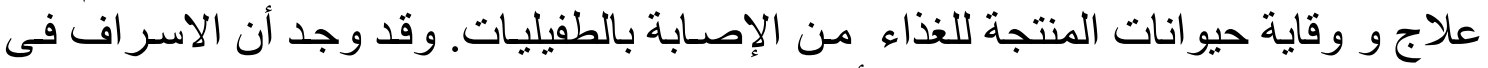

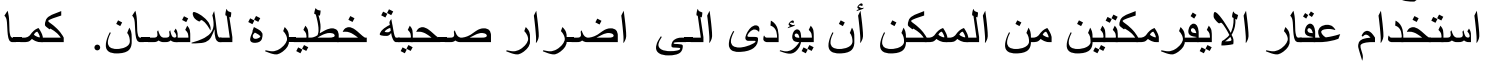

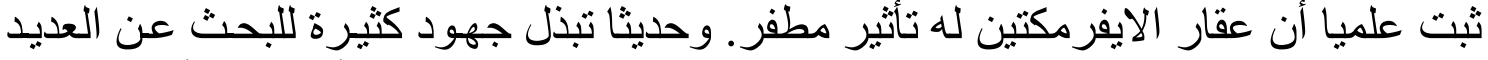

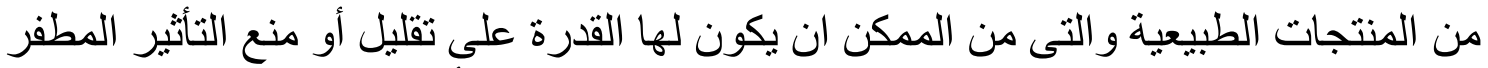

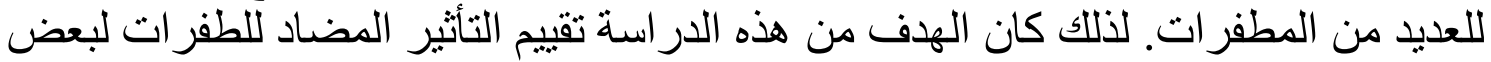

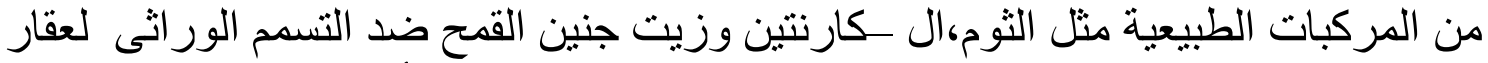

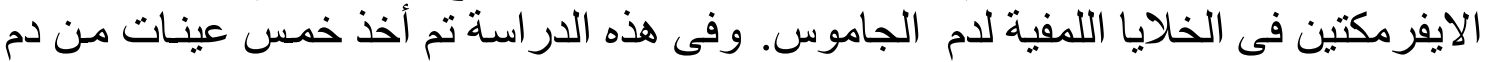

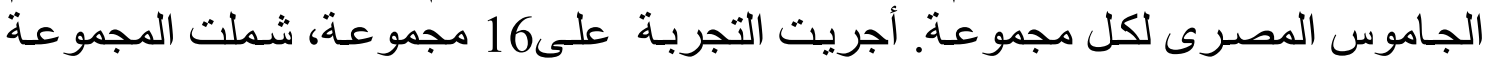

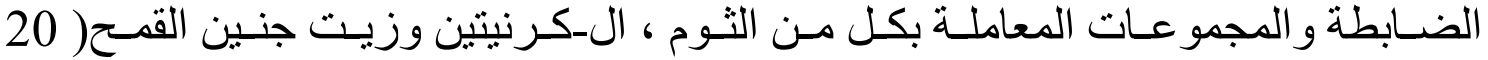

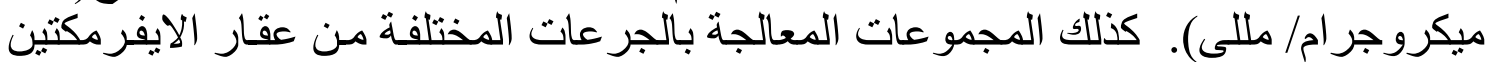

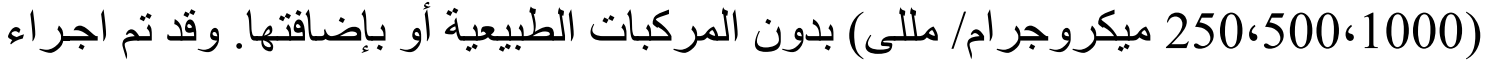
اختبار Cytokinesis block micronucleus assay لكل المجمو عات لتقدير معدلات الانوية الصغيرة باستخدام مادة السيتوكلازين-ب وكذلك تم تحضير الكروموسومات لتقدير معدلات

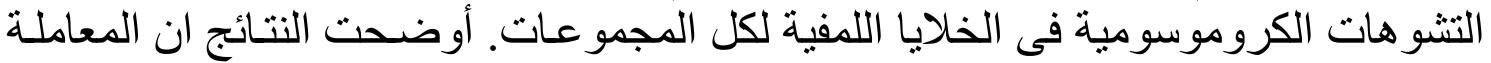

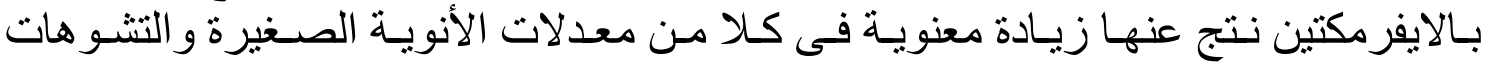

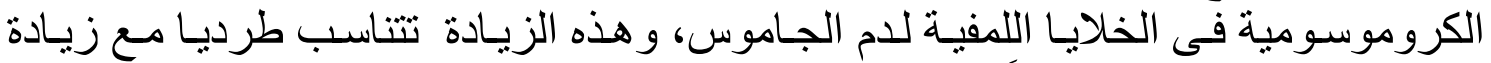

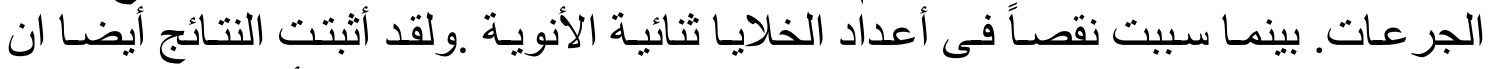

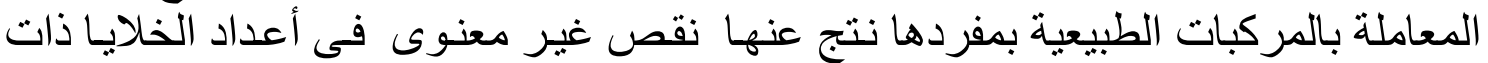

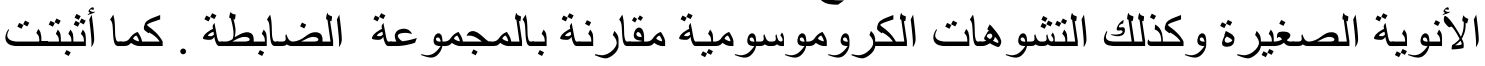

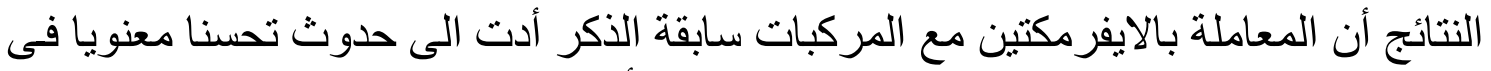

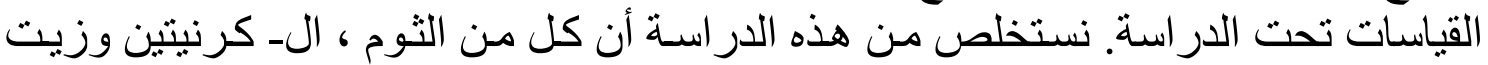
جنين القمح لها تأثير محسن فعال ضد السمية الور اثية الناتجة عن عقار الايفر التهن مكنين . 